Case Report

\title{
Rapid Development of Bleeding Esophageal Varices after Placement of Continuous Flow Left Ventricular Assist Device
}

\author{
Kaushal Majmudar (D), ${ }^{1}$ Michael Northcutt, ${ }^{2}$ Robert Gordon, ${ }^{3}$ and Claus J. Fimmel ${ }^{2}$ \\ ${ }^{1}$ Department of Medicine, Division of Internal Medicine, NorthShore University HealthSystem, Evanston, IL, USA \\ ${ }^{2}$ Department of Medicine, Division of Gastroenterology, NorthShore University HealthSystem, Evanston, IL, USA \\ ${ }^{3}$ Department of Medicine, Division of Cardiology, NorthShore University HealthSystem, Evanston, IL, USA
}

Correspondence should be addressed to Kaushal Majmudar; ksmajmudar@gmail.com

Received 18 July 2019; Accepted 10 September 2019; Published 6 November 2019

Academic Editor: Pier Cristoforo Giulianotti

Copyright ( 2019 Kaushal Majmudar et al. This is an open access article distributed under the Creative Commons Attribution License, which permits unrestricted use, distribution, and reproduction in any medium, provided the original work is properly cited.

We describe a patient with compensated cirrhosis and portal hypertension who underwent continuous flow LVAD implantation. Shortly after LVAD implantation, the patient developed new onset bleeding esophageal varices and ultimately had a fatal outcome. Our experience suggests that even well-compensated cirrhotic patients with significant portal hypertension are at risk of variceal bleeding after LVAD placement.

\section{Introduction}

Upper gastrointestinal bleeding-typically due to arterio-venous malformations-is common in patients with continuous flow left ventricular assist device (CF-LVAD), and is due to acquired von Willebrand disease and systemic anticoagulation [1-3]. We present a patient with compensated cirrhosis in whom large esophageal varices developed within a few weeks of LVAD placement, resulting in hemorrhage and eventual fatal outcome.

\section{Case Presentation}

A 62-year old male with a history of nonischemic cardiomyopathy presented with recurrent pulmonary edema requiring intra-aortic balloon pump (IABP) to maintain stability. On admission, EF was $19 \%$ and cardiac catheterization demonstrated no coronary artery disease. Given his recurrent pulmonary edema, a workup for CF-LVAD placement was initiated.

An abdominal CT scan unexpectedly revealed cirrhotic liver changes. The patient had no physical exam stigmata of advanced liver disease. Albumin, INR, transaminases, ferritin, and bilirubin were normal, and the platelet count was 170,000. Viral hepatitis serologies were negative. Upper GI endoscopy revealed no esophageal varices (Figure 1). A transjugular liver biopsy performed with the patient on IABP support, revealed a right atrial pressure of $0 \mathrm{mmHg}$ and hepatic vein wedge pressure of $21 \mathrm{mmHg}$, with a portal-systemic gradient of $20 \mathrm{mmHg}$. The liver histology confirmed cirrhosis without specific etiology. The patient's Child-Pugh class A was consistent with compensated cirrhosis. He underwent uneventful placement of a HeartWare ${ }^{\mathrm{TM}} \mathrm{HVAD}^{\mathrm{TM}}$ system (HeartWare, Framingham, MA). The patient was bridged from heparin to warfarin and started on aspirin. Seventeen days after LVAD placement, the patient sustained an acute upper GI bleeding episode with a drop of the hemoglobin from 8.6 to $4.9 \mathrm{~g} / \mathrm{dL}$. At the time, his INR was 2.4, and the platelet count 188,000. Emergent EGD revealed large, actively bleeding esophageal varices (Figure 2). Esophageal variceal band ligation was performed and hemostasis was achieved. An echocardiogram showed normal right ventricular function with no signs of right heart dilation or right heart failure. A Doppler abdominal ultrasound demonstrated patient hepatic veins with no evidence of Budd-Chiari. Over the following days, the patient developed ascites, hepatic encephalopathy and renal failure.

Twelve days later, the patient developed recurrent GI bleeding. EGD revealed active bleeding in the esophagus and a large clot. No specific bleeding source could be identified, and endoscopic attempts at hemostasis were unsuccessful. 


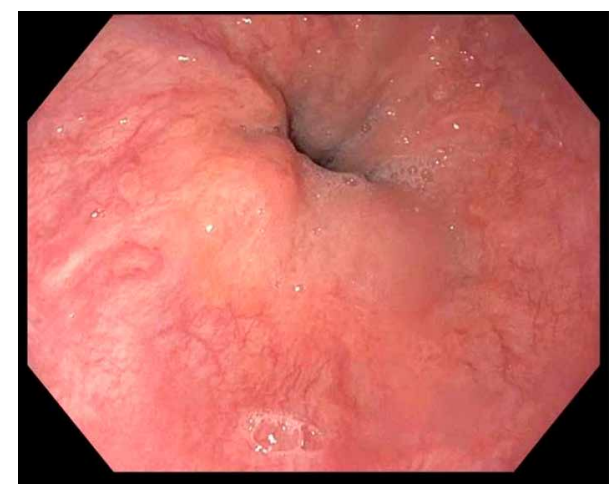

Figure 1: Normal gastroesophageal junction prior to CF-LVAD placement. No evidence of esophageal varices 4 days prior to LVAD placement.

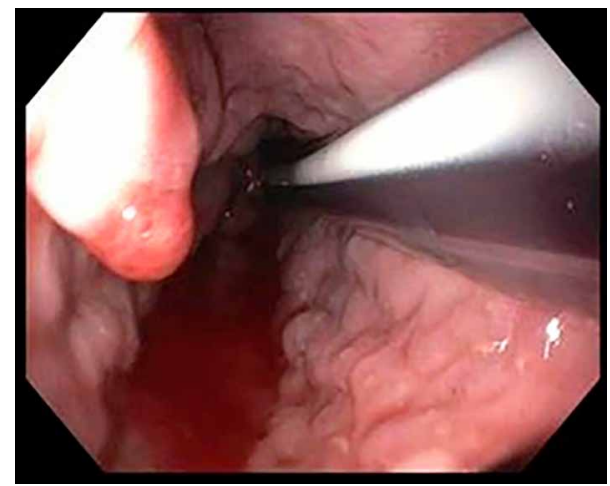

(a)

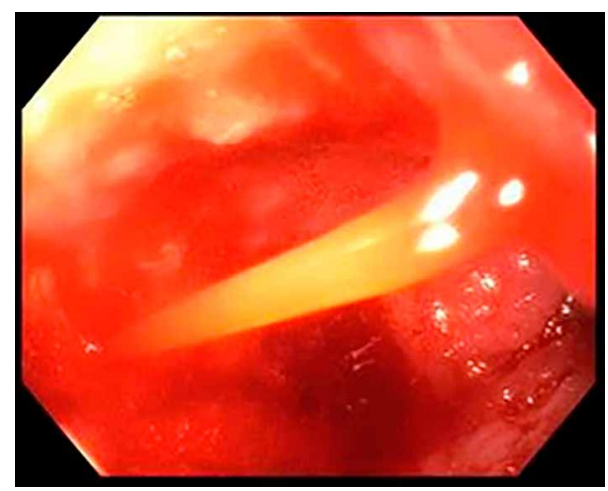

(b)

FIGURE 2: New onset esophageal varices 17 days after CF-LVAD placement. (a) Red wale sign with multiple esophageal varices in distal esophagus. (b) Active bleeding from new onset esophageal varices.

Placement of a transjugular, intrahepatic portosystemic shunt was considered, but the patient's MELD score of 40 was prohibitively high. The patient continued to bleed despite maximal medical support, and he expired on the following morning.

\section{Discussion}

We hypothesize that the fluid shifts and increased hepatic arterial inflow following LVAD placement caused rapid increases in esophageal variceal pressures and variceal bleeding in our patient. His low cardiac output prior to LVAD placement, likely masked the extent of his portal hypertension. In hindsight, the markedly elevated pre-LVAD transhepatic venous pressure gradient was the clearest predictor of postLVAD variceal complications in this patient. In addition, his moderately elevated MELD score of 11 prior to CF-LVAD placement suggested a moderately elevated risk for hepatic decompensation, despite a favorable Child-Pugh score [4].

We considered the possibility that the patient's portal hypertension became more severe as a result of right heart failure and acute congestive hepatopathy after CF-LVAD implantation. This is a relatively common complication that occurs in $28 \%$ of CF-LVAD patients a median of 14 days after placement [5]. However, the normal echocardiogram on the day of his first bleeding episode argues against this possibility.

Considering the significant resource requirements and medical risks of LVAD programs, judicious patient selection is critical. No published LVAD guidelines explicitly rule out the selection of all patients with cirrhosis [6]. Our experience suggests that even compensated cirrhotic patients with significant portal hypertension are at risk for variceal bleeding after LVAD placement.

\section{Conclusion}

Patients with compensated cirrhosis and significant portal hypertension are at risk for variceal bleeding after continuous flow LVAD placement. Pre-LVAD transhepatic venous pressure gradient may be a predictor of post-LVAD complications in a patient with compensated cirrhosis.

\section{Disclosure}

The abstract was presented at the Annual Scientific Meeting and Post Graduate Course of the American College of Gastroenterology in 2019.

\section{Conflicts of Interest}

The authors declare that they have no conflicts of interest.

\section{References}

[1] S. Islam, C. Cevik, R. Madonna et al., "Left ventricular assist devices and gastrointestinal bleeding: a narrative review of case reports and case series," Clinical Cardiology, vol. 36, no. 4, pp. 190-200, 2013.

[2] Z. T. Demirozu, R. Radovancevic, L. F. Hochman et al., "Arteriovenous malformation and gastrointestinal bleeding in patients with the HeartMate II left ventricular assist device," The Journal of Heart and Lung Transplantation, vol. 30, no. 8, pp. 849-853, 2011.

[3] V. M. Kushnir, S. Sharma, G. A. Ewald et al., "Evaluation of GI bleeding after implantation of left ventricular assist device," Gastrointestinal Endoscopy, vol. 75, no. 5, pp. 973-979, 2012. 
[4] S. Bruno, M. Zuin, A. Crosignani et al., "Predicting mortality risk in patients with compensated $\mathrm{HCV}$-induced cirrhosis: a long-term prospective study," The American Journal of Gastroenterology, vol. 104, no. 5, pp. 1147-1158, 2009.

[5] N. Yuan, G. J. Arnaoutakis, T. J. George et al., "The spectrum of complications following left ventricular assist device placement," Journal of Cardiac Surgery, vol. 27, no. 5, pp. 630-638, 2012.

[6] L. W. Miller and M. Guglin, "Patient selection for ventricular assist devices: a moving target," Journal of the American College of Cardiology, vol. 61, no. 12, pp. 1209-1221, 2013. 


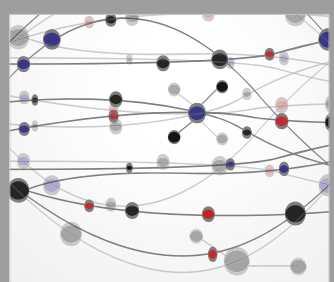

The Scientific World Journal
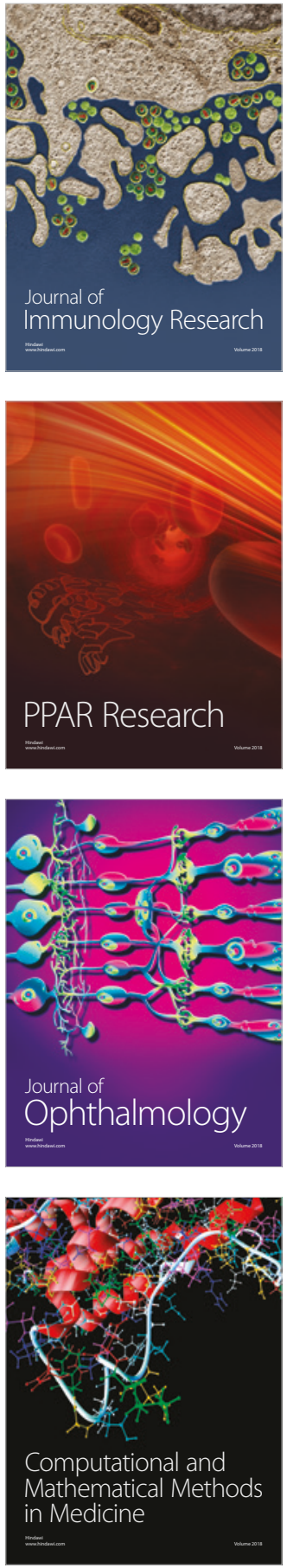

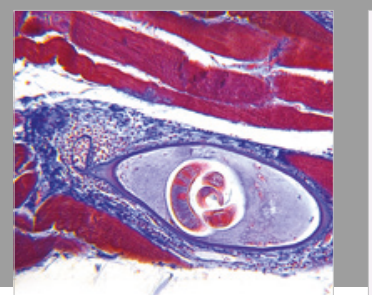

Gastroenterology Research and Practice

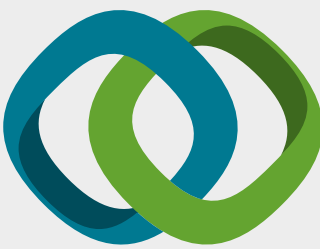

\section{Hindawi}

Submit your manuscripts at

www.hindawi.com
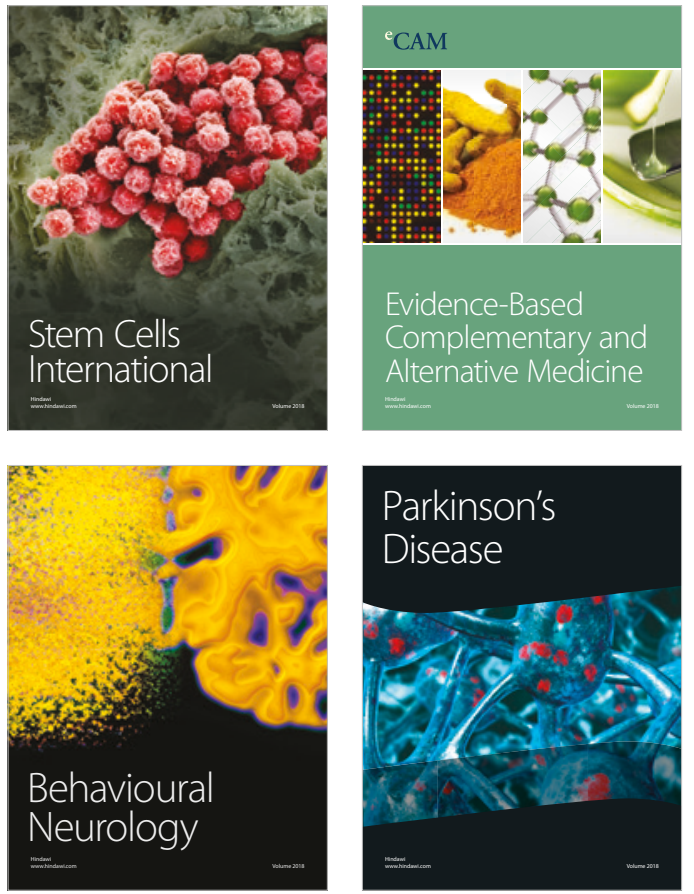

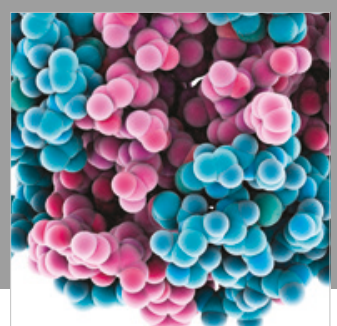

ournal of

Diabetes Research

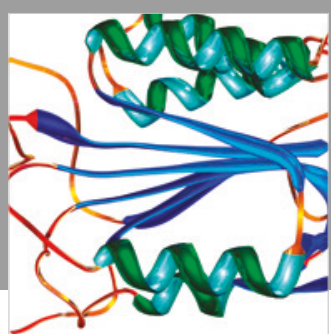

Disease Markers
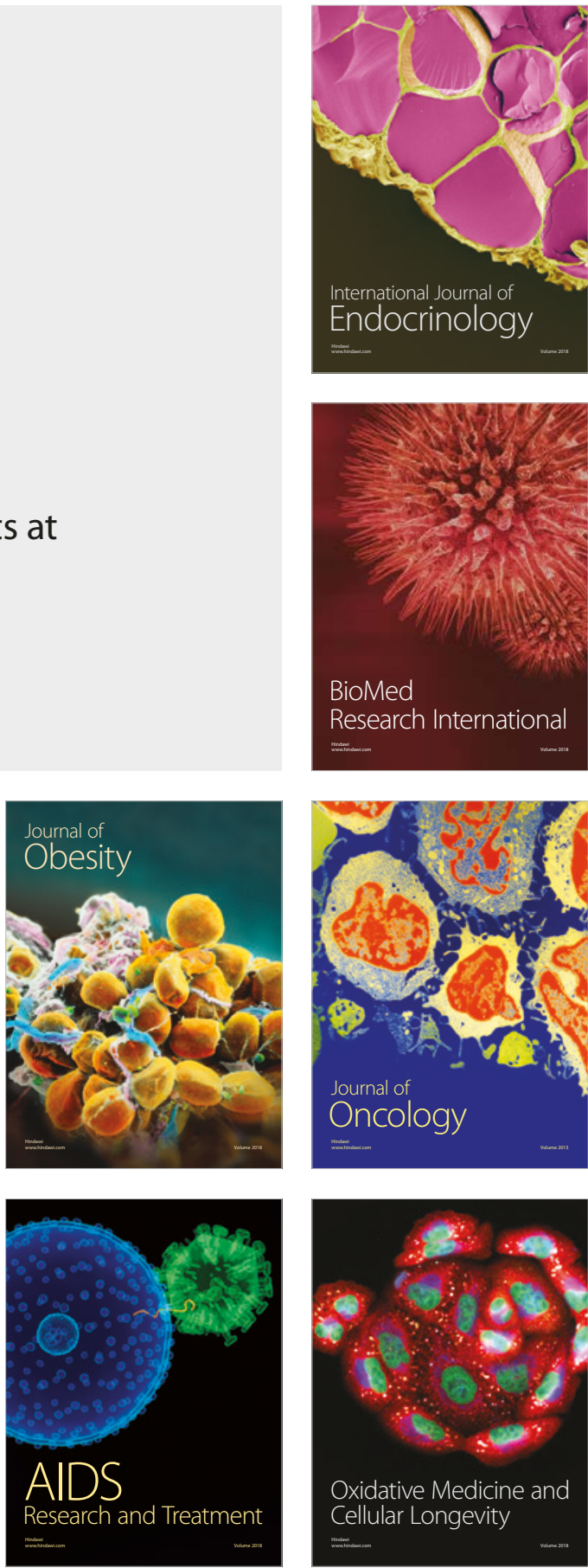